\title{
Biografie in cammino. Vite del Risorgimento e mitografie nazionali
}

\author{
Massimo Baioni*
}

\begin{abstract}
Biographies in Action. Lives of the Risorgimento and national mythologies. The debate about the Risorgimento has always been played out in the historiographical battlefield, as well as in the public arena. The article weaves together these two contexts by focussing on the genre of biography in all its manifestations. The first section looks at the exemplary case of Anita Garibaldi, from the various stages of her life to the images and representations of this heroine after her death. The second part analyses the great biographical dictionaries of the $20^{\text {th }}$ century which allow us to place the Risorgimento tradition within the context of Liberal, then Fascist and finally Republican Italy.
\end{abstract}

Key words: Italian Risorgimento, Anita Garibaldi, Biographies, Public Memory Parole chiave: Risorgimento, Anita Garibaldi, Biografie, Memoria pubblica

Sin dalla primissima stagione postunitaria, il genere biografico ha costituito un canale potente e seducente del racconto pubblico del Risorgimento. Oscillante tra agiografia, contestazione, torsioni municipalistiche, esso si è strutturato intorno a modalità che si sono protratte con poche varianti almeno fino al centenario del 1961, grazie anche alla diffusione che ne hanno garantito altri strumenti di circolazione, dai musei al cinema, dalla pittura alla narrativa. La produzione biografica non ha mancato di investire la sfera storiografica, che a sua volta non è rimasta impermeabile alle forti implicazioni politiche connesse allo studio del Risorgimento e al peso esercitato da un'accezione della storia che Gramsci vedeva declinata in termini di «biografia nazionale». Il flusso non si è di fatto arrestato all'indomani del centenario, anche quando si trattava di biografie sui generis, come il Cavour di Rosario Romeo; ma in quegli anni la predilezione degli storici stava ormai orientandosi in altre direzioni, trainata dalle discussioni sui limiti dello sviluppo e dal bisogno di colmare lacune gravi in tema di condizioni economiche e sociali, di agricoltura, industria, commercio nei decenni tra XVIII secolo e unità.

* Dipartimento di Studi storici (Milano), Via Festa del Perdono 720122 Milano; massimo.baioni@unimi.it. 
Dopo una fase di appannamento, la ricomparsa dell'approccio biografico che si registra nell'agenda degli studiosi del Risorgimento può essere ricondotta a una serie concomitante di fattori. In generale, si avverte forse anche in questo ambito una reazione alla tendenza a nascondere gli individui dietro categorie impersonali, a descrivere la realtà prevalentemente «attraverso anonimi rapporti di potere» ${ }^{1}$. Sul piano propriamente storiografico, hanno inciso a vario titolo il ritorno dell'"evento", il successo della storia culturale, l'attenzione posta sulla dimensione emozionale dell'azione politica, l'esplorazione della soggettività lungo le traiettorie di vita transnazionali e le complesse identità di confine ${ }^{2}$. "Storie" di uomini e donne beneficiano del recupero di fonti autobiografiche (epistolari, diari, memorie) e dell'intensa attività di istituzioni impegnate su questo terreno, che non sempre riesce a evitare un'enfasi scivolosa sul versante della memoria. I tanti anniversari che si sono succeduti negli ultimi anni e il riaccendersi della discussione politica sul passato, alimentata dalla visibilità crescente di un revisionismo cattolico e neoborbonico, hanno incoraggiato a loro volta il revival biografico. Restando in campo risorgimentale, e solo per citare alcuni esempi importanti, basti ricordare i volumi su Mazzini, Garibaldi, Cavour e la folta produzione innescata dal centocinquantenario del $2011^{3}$.

Se possiamo riconoscere nell'attuale fortuna del genere biografico la spia di una particolare sensibilità verso la storia dell' 800 italiano, occorre però aggiungere che esso va inteso in un'accezione ampia, come una sorta di contenitore che accoglie al suo interno approcci e sguardi di natura disparata. Non solo dunque le biografie classiche, le storie di vita di uomini e donne del Risorgimento, che sono oggi rivisitate alla luce dei nuovi filoni di ricerca e con un solido ancoraggio al nesso tra i percorsi in vita dei personaggi e le loro proiezioni nella memoria collettiva. A quel contenitore appartengono nondimeno le biografie famigliari ${ }^{4}$ e quelle delle "famiglie politiche" dell' 800 , dai "piccoli cospiratori" mazziniani ai garibaldini studiati rispettivamente da Arianna Arisi

\footnotetext{
${ }^{1}$ S. Loriga, La piccola x. Dalla biografia alla storia, Sellerio, Palermo 2012, p. 15.

${ }^{2}$ Cfr. M. Verginella, La guerra di Bruno. L'identità di confine di un antieroe triestino e sloveno, Carocci, Roma 2015.

${ }^{3}$ R. Sarti, Giuseppe Mazzini. La politica come religione civile, Laterza, Roma-Bari 2005; L. Riall, Garibaldi. L'invenzione di un eroe, Laterza, Roma-Bari 2007; M. Isnenghi, Garibaldi fu ferito. Storia e mito di un rivoluzionario disciplinato, Donzelli, Roma 2007; S. Levis Sullam, L'apostolo a brandelli. L'eredità di Mazzini tra Risorgimento e fascismo, Laterza, Roma-Bari 2010; A. Viarengo, Cavour, Salerno, Roma 2010; G. Belardelli, Mazzini, il Mulino, Bologna 2011.

${ }^{4}$ Un buon esempio di ricerca sul territorio, con una particolare sensibilità verso la storia del notabilato, viene dai volumi della collana del Comitato di Torino dell'Istituto per la storia del Risorgimento italiano, tra i quali segnalo almeno S. Cavicchioli, Famiglia, memoria, mito. I Ferrero della Marmora (1748-1918), Carocci, Roma 2004.
} 
Rota ed Eva Cecchinato5; ma vi possono confluire altresì le "biografie" dei monumenti che raccontano pezzi importanti della storia nazionale, quali il Vittoriano e la controversa statua di Giordano Bruno in Campo dei Fiori a Roma ${ }^{6}$.

Due contributi recenti, tra gli altri che potrebbero essere presi in esame ${ }^{7}$, aiutano a cogliere da angolazioni originali e con risultati di notevole interesse alcune peculiarità di questa fase degli studi. Dedicati rispettivamente ad Anita Garibaldi e a tre grandi dizionari biografici del '900, i libri di Silvia Cavicchioli e Maria Pia Casalena ${ }^{8}$ si collocano evidentemente su piani diversi in quanto a struttura, fonti, impostazione. Le loro differenti tipologie si prestano nondimeno a dar conto dell'eterogeneità degli sguardi tematici connessi al genere biografico e alla circolarità delle domande che esso solleva; soprattutto consentono di mettere in relazione la prospettiva individuale propria della biografia storica e i profili integrati nel corpus collettivo dei dizionari, valorizzandoli nel contesto dei processi di costruzione (e contestazione) delle mitologie patriottiche nelle macro stagioni storiche dell'Italia unita.

\section{Di Anita Garibaldi, in vita e in morte}

Il libro di Cavicchioli su Anita rientra solo apparentemente nel filone delle biografie tradizionali. Gli elementi di novità sono numerosi e scaturiscono dallo sforzo di ribaltare a fini esplorativi una presenza ingombrante come quella di Garibaldi, capace di oscurare tutti coloro che hanno gravitato nell'orbita della sua azione e del suo stesso mito. La brasiliana Ana Maria de Jesus Ribeiro da Silva è stata parte costituiva di quel mondo. La sua breve esistenza e specialmente i tempi e le modalità della morte ne hanno fatto un personaggio non meno leggendario del consorte, secondo una immagine oleografica consegnata al mito dalle Memorie di Garibaldi medesimo e dai primi biografi del generale (da Guerzoni a Dumas, da Bandi a Trevelyan). Cavicchioli ha dunque

5 A. Arisi Rota, I piccoli cospiratori. Politica ed emozioni nei primi mazziniani, il Mulino, Bologna 2010; E. Cecchinato, Camicie rosse. I garibaldini dall'Unità alla Grande Guerra, Laterza, Roma-Bari 2007.

${ }^{6}$ C. Brice, Il Vittoriano. Monumentalità pubblica e politica a Roma, Archivio Guido Izzi, Roma 2005; B. Tobia, L'Altare della Patria, il Mulino, Bologna 1998; M. Bucciantini, Campo dei Fiori. Storia di un monumento maledetto, Einaudi, Torino 2015.

${ }^{7}$ Ad es., nella collana Profili dell'editore Salerno, i volumi di M. Mascilli Migliorini, Metternich (2014); A. Viarengo, Cavour cit. e Id., Vittorio Emanuele II (2017).

${ }^{8}$ S. Cavicchioli, Anita. Storia e mito di Anita Garibaldi, Einaudi, Torino 2017; M.P. Casalena, Eroi in bilico. Il Risorgimento nei dizionari biografici del Novecento, Carocci, Roma 2018. 
fondate ragioni per fare di Anita il fulcro di una ricostruzione autonoma, in grado di affrancarla da una dipendenza che è stata comune a tante patriote e figure femminili del Risorgimento «passate alla storia di riflesso, in posizione marginale rispetto a quella di compagni, figli, fratelli» (p. VIII) .

Lo stesso mito di Anita, pur inestricabilmente connesso a quello garibaldino, si distingue per caratteristiche proprie, che consentono di investigare in modo non scontato alcuni percorsi memoriali anche secondo un'ottica di genere. L'ampio spazio riservato alla memoria di Anita, l'individuazione dei suoi aspetti caratterizzanti e degli snodi temporali che ne segnano il rapporto con i diversi contesti politici e culturali inseriscono solidamente il volume anche nel campo degli studi sui linguaggi e le pratiche dei processi di nation building.

Abbinando rigore di metodo a una scrittura fluida, Cavicchioli tesse una trama avvincente, dentro la quale si muovono storie, personaggi, eventi che straordinari lo sono stati realmente. La prima sezione del libro segue Anita "in vita", dalle notizie sull'infanzia all'incontro con Garibaldi nel 1839, dalle battaglie sudamericane all'epilogo italiano del 1848-49. Anita emerge come una giovane donna che, dopo essersi unita in matrimonio, appena quattordicenne, con il calzolaio Manoel Duarte de Aguiar, trae dall'incontro con Garibaldi la forza per prendere in mano il proprio destino. Un dato controverso, questo dell'"adulterio", che insieme ad altri avrebbe pesato non poco sull'immagine di immoralità coniata a più riprese dai detrattori di Garibaldi. Di fatto, il carattere ribelle e autonomo è il filo conduttore della personalità di Anita: e si conferma con forza quando, approdata con i figli a Nizza nel marzo 1848, decide di sacrificare il ruolo di madre a quello di donna che intende condividere la battaglia e le idee del compagno. Così facendo, Anita «rinunciava all'adempimento degli obblighi familiari, al ruolo tradizionale femminile riconosciuto alle donne nell'Ottocento, quello materno e domestico, abdicava al modello muliebre simbolicamente più forte, quello di madre, per quello di donna libera ed emancipata, che sceglieva di amare, di essere accanto al marito nel tormento dell'azione» (p. 45). Tale decisione si manifestò nelle sue implicazioni più drammatiche nei giorni finali dell'assedio francese alla repubblica romana $\mathrm{e}$ poi della perigliosa marcia verso Venezia. Le cronache e le testimonianze di quanti ebbero modo di vederla in quelle settimane ${ }^{10}$ consentono a Cavicchioli di spogliare Anita degli abbellimenti della mitologia romantica, che ha esteso

9 Cfr. S. Soldani, Il Risorgimento delle donne, in Storia d'Italia, Annali, 22, Il Risorgimento, a cura di A.M. Banti e P. Ginsborg, Einaudi, Torino 2007, pp. 183-224; A. Tafuro, Madre e patriota. Adelaide Bono Cairoli, Firenze UP, Firenze 2011.

${ }^{10} \mathrm{La}$ fonte principale in questo caso è il lungo lavoro sul campo compiuto da R. Belluzzi, La ritirata di Garibaldi da Roma, Società editrice Dante Alighieri, Roma 1899. 
anche all'ultimo mese della sua vita $i$ tratti improbabili dell'intrepida combattente: la donna viene mostrata nella sua condizione di stanchezza e sofferenza, esausta a causa dei continui spostamenti, spossata dalla gravidanza e dai sintomi della febbre malarica che la porterà alla morte, non piegata tuttavia dalle avversità.

La cornice dell'epilogo, nei primi giorni di agosto, è quella destinata a lasciare il segno più forte nell'immaginario patriottico. Dopo lo sbarco a Magnavacca, Ciceruacchio e i figli sono catturati dagli austriaci e fucilati, seguiti nella stessa sorte a distanza di pochi giorni da Ugo Bassi e Giovanni Livraghi. Braccato e rimasto solo con Anita morente e con Leggero, Garibaldi assiste il 4 agosto al decesso della moglie nella fattoria Guiccioli. Deve però affidare ad altri il compito di seppellirla, trascinato via dai soccorritori, poi celebrati come i suoi "salvatori", artefici della celebre "trafila" tra le valli di Comacchio, Ravenna e l'Appennino, grazie alla quale Garibaldi può eludere la caccia austriaca e approdare dopo un lungo peregrinare alle coste del Tirreno.

Inizia da qui la seconda parte della storia di Anita: quella che ne proietta la morte tragica sul versante della memoria, facendone un tassello importante della mitologia risorgimentale e garibaldina. Su questo terreno il libro porta un contributo ricco e articolato. I canali e i linguaggi della integrazione di Anita nello spazio pubblico e nei circuiti memoriali diventano chiavi di lettura preziose per cogliere fenomeni più generali. Tra questi, la centralità del corpo come catalizzatore di significati simbolici, i modelli di rappresentazione dell'eroina, la prolungata pregnanza del canone martirologico e delle reliquie dei combattenti per l'indipendenza: tutti aspetti che ebbero in Garibaldi un regista attento, lucido e consapevole della posta in gioco. La rilevanza del corpo affiora sin dalla controversa dissepoltura delle spoglie di Anita, quando fu diffusa ad arte la leggenda nera di un decesso accelerato da uno strangolamento da parte di Garibaldi. Il processo di personale elaborazione del lutto si snodò lentamente e si concluse soltanto nel settembre 1859, con il recupero della salma di Anita e la sua traslazione a Nizza. Sin dall'esilio nordamericano, Garibaldi curò personalmente, con «atteggiamento protettivo», la gestione dell'immagine di Anita, disegnando il doppio profilo di donna coraggiosa e virtuosa, dolente e vittima (p. 90). Partendo dai ricordi manoscritti di Garibaldi, sulla cui natura di documento-monumento oggetto di varie redazioni e di continue revisioni Cavicchioli scrive pagine ben sorvegliate, il libro si sofferma sul modello di eroina che fu elaborato mediante scritti, dipinti, monumenti, celebrazioni pubbliche. Oltre al ruolo di Jessie White Mario, sarebbe stato interessante introdurre qui un confronto più denso con la tradizione storiografica declinata al femminile, in modo da fare risaltare le dinamiche che si fecero strada nella 
riflessione sul passato tramite la lente di genere ${ }^{11}$. Mentre in un primo tempo la biografia di Anita fu assorbita pressoché interamente dalla vicenda della morte e «incastonata nel momento sacrificale» (p. 107), con il passare degli anni la lettura si allargò e sfociò nella «raffigurazione idealtipica dell'eroina romantica» (p. 150). Decisivo in questo caso risultò l'apporto di Dumas, che inserì nella narrazione varie licenze letterarie e molteplici «effetti di drammatizzazione» (p. 148), enfatizzando tutti gli ingredienti del romanticismo politico, dal coraggio femminile al ruolo della coppia eroica in cui il legame sentimentale si salda all'amore di patria. Sono stilemi che ritornano con frequenza nella produzione teatrale dell'epoca ${ }^{12}$ e che meriterebbero di essere analizzati anche con riferimento alle prime produzioni cinematografiche, tra cui spicca il cortometraggio Anita Garibaldi di Mario Caserini (1910).

Come per altri eventi e personaggi del Risorgimento, alcuni luoghi della memoria costituiscono osservatori di particolare utilità per misurare le dinamiche di circolazione del mito di Anita e i suoi livelli di radicamento popolare, ben certificati persino dalle occorrenze onomastiche. Non sorprende che il culto abbia toccato proporzioni notevoli in Romagna: qui i segni del passaggio di Anita - e di Garibaldi, ovviamente - configurarono rapidamente una geografia simbolica in grado di alimentare i rituali, i pellegrinaggi patriottici e il senso di appartenenza delle robuste culture politiche democratiche e repubblicane. Durante l'anniversario del 1907, Anita fu pienamente coinvolta nella contesa che contrappose le diverse anime della memoria risorgimentale, e specialmente il mondo cattolico sfruttò l'occasione per incrinare la moralità garibaldina, rilanciando la polemica sul primo matrimonio e sulle circostanze delle morte.

Una sintesi "normalizzatrice" fu tentata infine dal fascismo, anche se l'operazione volta ad additare Anita come modello per le giovani italiane non si presentava né facile né scontata, alla luce dei tratti di evidente anticonformismo del personaggio. Lo sforzo raggiunse il punto di massima visibilità nel 1932, nell'ambito delle cerimonie per il cinquantesimo anniversario della morte di Garibaldi. In quell'occasione celebrativa ad Anita fu riservato un posto di primaria importanza. Da Nizza e poi da Staglieno le sue ceneri, rivendicate invano anche dal Brasile del dittatore Gétulio Vargas, furono trasferite a Roma e qui tumulate alla base del monumento equestre inaugurato da Mussolini il 4 giugno. L'opera di Mario Rutelli rovesciava l'immagine classica: Anita viveva

\footnotetext{
${ }^{11}$ Cfr. ad es. I. Porciani, Les historiennes et le Risorgimento, «Mélanges de l'École Française de Rome. Italie et Méditerranée», 112 (2000), n. 1, pp. 317-57.

12 Cfr. C. Sorba, Il melodramma della nazione. Politica e sentimenti nell'età del Risorgimento, Laterza, Roma-Bari 2015.
} 
di vita propria, tornava ad essere l'amazzone guerriera e indomita con la pistola in pugno, non più cristallizzata nell'attimo sacrificale dell'epilogo. Il suggerimento di Mussolini di aggiungere il piccolo Menotti nel braccio sinistro consentiva di recuperare il ruolo materno, ingrediente indispensabile a supporto della visione fascista della donna e della campagna demografica, temi cari anche alla Chiesa da poco riconciliatasi con lo Stato. Benché non assenti nei vari rivoli della cultura fascista, le letture poco ortodosse dovettero cedere quasi sempre il passo. Nel volume Garibaldi (1941), ospitato nella collana I grandi italiani diretta da Luigi Federzoni per la Utet, Aldo Valori non esitava a discostarsi dall'immagine rivoluzionaria ed emancipatrice della donna, che riconduceva dentro il recinto rassicurante di una «missione umana e sociale» di stampo conservatore $^{13}$.

Il libro di Cavicchioli si conclude con il richiamo all'inaugurazione nel 1939 del villaggio rurale dedicato ad Anita, a pochi chilometri dai luoghi che nell'estate 1849 erano stati lo scenario della sua morte e del trafugamento di Garibaldi. La scelta taglia fuori alcuni passaggi non secondari, che avrebbero potuto dare alla narrazione una periodizzazione ancora più compiuta e coerente con gli assunti di partenza. Il nome di Anita non uscì indenne dalla durissima contrapposizione tra partigiani e fascisti di Salò che investì la memoria del Risorgimento nei mesi della guerra civile. Le questioni si riaffacciarono in tutta la loro rilevanza politica e simbolica all'indomani della liberazione. Il giovane Stato democratico, ben presto immerso nelle vischiosità della guerra fredda, non esitò a recuperare ogni rivolo della cultura democratica e repubblicana del Risorgimento al fine di proiettare sulle nuove istituzioni il retaggio della tradizione patriottica. Mi pare dunque che l'epilogo naturale di un secolo di rammemorazioni e immagini di un personaggio femminile presente così a lungo nello spazio pubblico si possa individuare proprio nella breve ma densa fase che intercorre tra la nascita della Repubblica e il centenario della morte di Anita. Dibattiti in Parlamento e nella stampa, retoriche discorsive, organizzazione e geografia delle celebrazioni, rappresentazioni cinematografiche - in particolare Cavalcata d'eroi (1949) e Camicie rosse (1952), con Raf Vallone e Anna Magnani nei panni di Garibaldi e Anita ${ }^{14}$-, travasi dei modelli della biografia

13 Cfr. A. Pedio, Le collane editoriali di storia. Da «I Prefascisti» a «I Grandi Italiani», in Storiografia, cultura storica e circolazione del sapere nell'Italia fascista, a cura di M. Angelini e M. Carrattieri, «Storiografia», 9 (2005), pp. 199-200.

14 Per considerazioni generali cfr. P. Cavallo, Storia, cinema e identità nazionale (1932-1962), Liguori, Napoli 2009; T. Giardina, Schermi multipli e plurime visioni. La grande Madre. L'Italia, Marsilio, Venezia 2015. 
commemorativa e dell'immaginario femminile tra Risorgimento e Resistenza ${ }^{15}$ : sono tutti ambiti e tematiche che meriteranno utili supplementi di ricerca, sulla scia delle numerose suggestioni offerte dal bel libro di Silvia Cavicchioli.

\section{Documenti-monumenti. I grandi dizionari biografici}

Se l'intreccio tra storia e memoria, esistenze individuali e vicende nazionali (o transnazionali), rappresentazioni di genere, usi pubblici del passato risulta ampiamente documentato nel caso di una biografia storica esemplare come quella di Anita Garibaldi, esso assume implicazioni ancora più vaste quando si passi a investigare l'impianto e gli obiettivi di operazioni per loro natura estremamente complesse come i dizionari biografici.

In questo senso, il libro di Maria Pia Casalena ha il merito di estendere ulteriormente il perimetro delle tematiche e dei metodi di indagine insiti nell'approccio biografico. Il suo lavoro assume alcuni grandi dizionari a cartine di tornasole di temperie storiografiche, diatribe politiche e culturali che hanno attraversato l'Italia del ' 900 . Mettendo a frutto la frequentazione delle autobiografie nel lungo ' 800 , del Risorgimento e di alcune sue ricadute nella memoria pubblica ${ }^{16}$, l'autrice dissoda con perizia un terreno che si rivela molto fruttuoso. La vicenda delle voci biografiche risorgimentali diventa «una faccia eloquente, nel suo piccolo, della storia del nostro paese e della guerra dei nation building che si è combattuta in ogni stagione» (p. 177), utile a comprendere anche i più recenti e vistosi tentativi volti non solo a demolire le gallerie degli antenati, ma a ribaltarne le gerarchie di eventi, uomini, valori. In ordine cronologico, il libro esordisce con l'impresa diretta da Michele Rosi per Vallardi - Il Dizionario del Risorgimento Nazionale -, ideata in età giolittiana ma completata e pubblicata solo negli anni '30, dopo la morte di Rosi $(1934)^{17}$. La sua lunga e contrastata vicenda corre parallela a un certo punto con due altre significative iniziative editoriali del ventennio fascista. La prima, coordinata da Mario Menghini (il curatore dell'Opera omnia di Mazzini) ma rimasta sulla carta, fu concepita nell'orbita dell'Enciclopedia italiana e respirò l'influenza gentiliana. La seconda, gestita dall'ex ministro dell'Educazione nazionale

\footnotetext{
15 Alcune indicazioni in A.R. Perry, Il santo partigiano martire. La retorica del sacrificio nelle biografie commemorative, Longo, Ravenna 2001.

16 M.P. Casalena, Biografie. La scrittura delle vite in Italia tra politica, società e cultura (1796-1915), Bruno Mondadori, Milano 2012; Ead., Controstorie del Risorgimento. Dal locale al nazionale (2000-2011), «Memoria e Ricerca», 20 (2012), n. 40, pp. 163-82; Ead. (a cura di), Antirisorgimento. Appropriazioni, critiche, delegittimazioni, Pendagron, Bologna 2013.

17 Vallardi, Milano 1931-1937.
} 
Francesco Ercole e in parte erede del lemmario avviato da Menghini, sfociò nella pubblicazione alla fine degli anni '30 dell'Enciclopedia biografica $e$ bibliografica italiana. Il Risorgimento italiano (I martiri; Gli uomini politici; I combattenti $)^{18}$. La ricerca di Casalena approda infine alle voci risorgimentali (limitatamente alle lettere A-C) incluse nei primi 31 volumi del Dizionario Biografico degli Italiani, usciti tra 1960 e 1986 durante la direzione di Alberto Maria Ghisalberti ${ }^{19}$ : un contenitore, quest'ultimo, ben diverso dai precedenti, dove il Risorgimento non è più immediatamente riconoscibile nella sua specificità e i lemmari degli italiani meritevoli di ricordo sono diluiti in quindici secoli di storia.

Il confronto tra i dizionari fa affiorare le strategie che nei diversi contesti hanno presieduto al racconto del Risorgimento, le continuità e le rotture, le circostanze della fortuna e della sfortuna di protagonisti, comprimari, antagonisti del processo dell'unità italiana. I rimodellamenti, lungi dal riguardare soltanto il Risorgimento come fenomeno generale, non risparmiarono infatti «le stesse traiettorie individuali», che furono in perenne bilico e subirono «aggiustamenti, metamorfosi, stravolgimenti alla luce delle esigenze dell'opera e del momento» (p. 102). Si mescolano così fattori di lungo periodo, forzature ideologiche, sforzi di ricomposizione dell'insieme in una chiave scientificamente affidabile, esigenza che si fa strada specialmente con il varo del $D B I$ all'inizio degli anni '60. In una densa prospettiva di sintesi e con capitoli ben organizzati, sono tenute insieme questioni che intrecciano controversie storiografiche, strategie discorsive, dinamiche editoriali, gerarchie territoriali, orientamenti politici. Da qui la scelta di non isolare i singoli segmenti temporali e di collocarli il più possibile in dialogo reciproco tramite l'analisi incrociata di alcune domande e tematiche che si ritrovano all'origine di ogni dizionario. Il continuo movimento in avanti e indietro sulla linea del tempo, se può talora rallentare la fluidità narrativa, si rivela in realtà lo schema adeguato per dare spessore agli interrogativi di partenza e per valorizzare le implicazioni connesse a quelle imprese editoriali.

D'altronde, sarebbe vano rinvenire nei dizionari tragitti lineari, coerenza assoluta tra obiettivi e risultati, assenza di contraddizioni e financo di esiti inaspettati. Accanto alla volontà di "ordinare" il passato, il dizionario reca in sé paradossalmente - un "disordine" che deriva proprio dalla struttura alfabetica (circa 9.000 voci nel Rosi, che diventano 12.000 nell'Ercole) e dalla varietà delle variabili che entrano in gioco nella sua ideazione e realizzazione. Un dizionario

\footnotetext{
${ }^{18}$ Editrice Bibliografica italiana, Roma 1939-45.

${ }^{19}$ Istituto della Enciclopedia Italiana, Roma 1960-1986.
} 
non è il frutto di un'operazione neutrale e asettica, come sottolinea più volte Casalena, che raccoglie le note di metodo di Jean-Luc Chappey ${ }^{20}$ e le filtra anche con un uso attento delle corrispondenze tratte dagli archivi. I collaboratori contattati per redigere le voci, il controllo e l'intervento dei direttori, la scelta dei soggetti biografati e parimenti i nomi degli esclusi, la dimensione dei lemmi, la tipologia delle bibliografie, i tempi di realizzazione del progetto, che finiscono per risentire dei forti mutamenti di contesto, storiografici e ancor più politici: tutto ciò, se osservato in prospettiva e sul terreno del confronto incrociato tra le iniziative editoriali in questione, porta in superficie visioni diverse del passato, genealogie rivendicate o contrastate, rende i dizionari una lente preziosa per cogliere l'importanza loro assegnata anche nel tratteggiare un profilo del presente e del futuro.

Questo itinerario, in cui si legano individuale e collettivo, nazionale e locale, percorsi generazionali di studiosi appartenenti a varie scuole storiografiche, viene esplorato in quattro capitoli (Inventari del patriottismo, Dal generale al particolare, Metamorfosi biografiche, Italia in cammino), ciascuno dei quali rinvia ai nodi cruciali selezionati dall'autrice per dipanare una matassa decisamente intricata. Dall'analisi emerge quanto fosse viva la consapevolezza che strumenti come i dizionari potessero incidere nella formazione della memoria nazionale, orientare studiosi nelle loro ricerche e al tempo stesso cristallizzare immagini di patrioti nel sentimento comune. Il dosaggio di presenze e assenze, le gerarchie di rilevanza e la chiave di lettura adottata assumevano così un peso strategico decisivo. Casalena porta conferme molteplici e convincenti di questo assunto. Impostato nella fase finale del giolittismo e nella temperie del conflitto e del dopoguerra, il dizionario Rosi si caratterizzò per una narrazione vicina alle specificità territoriali e per una "difesa" del Risorgimento che risentiva delle posizioni cattolico-liberali anti temporaliste (e già neutraliste) del suo direttore. Quando uscirono i primi volumi, all'inizio degli anni '30, esso sembrava ormai «un monumento fuori dal tempo, che assecondava un eroismo non più di moda, che si appoggiava a libri "superati", che - soprattutto - insisteva nel decantare un'Italia liberale dalle nobili origini e un "popolo" [...] che non assomigliava in nulla a quello "martire e marziale" teorizzato e cantato da tanta storiografia sul Risorgimento apparsa tra Concordato e Patto d'Acciaio» (p. 62). In effetti, l'operazione intrapresa da Ercole, «colossale lapide di carta, volta a commuovere più che a ricordare» (p. 81), siglava un mutamento evidente di impostazione e linguaggio. Formato da

20 J.-L. Chappey, Ordres et désordres biographiques. Dictionnaires, listes de noms, réputations des Lumières à Wikipedia, ChampVallon, Seyssel 2013. 
voci brevi, con l'obiettivo di una completezza che finiva per penalizzare lo scavo biografico, il dizionario Ercole respirava gli effetti dell'alleanza con la Germania nazista e le ambizioni imperiali dell'Italia fascista, secondo una diffusa tendenza alla militarizzazione della storia e della memoria ${ }^{21}$. Ciò che contava era la collocazione del passato all'interno di una griglia di uomini (in primis i combattenti e i martiri), categorie e valori che prefiguravano un presente scolpito dall'esperienza della guerra, del milite ignoto e dello squadrismo. Non a caso, l'esclusione investiva tre categorie ben definite - le donne, gli ebrei, gli stranieri -, le quali avevano ricevuto viceversa un'attenzione tutt'altro che marginale nel Rosi. La voce su Cavour, più ancora di quelle degli altri "grandi fattori" dell'unità, era lo specchio esemplare delle differenze di fondo sull'idea di patria e di nazione che separavano i due dizionari. Per Rosi, che pure - precisa Casalena - non era stato «un paladino della dialettica parlamentare» (p. 128), l'eroismo del conte e la superiorità della sua visione si riassumevano nella modernità del pensiero e dell'azione politica; la rilettura fascista dei titoli patriottici di Cavour ne decretava viceversa la subalternità a Mazzini e Garibaldi, più malleabili sul piano ideologico e dell'uso pubblico ${ }^{22}$. Tramontata la vena martirologica e attenuatasi all'inizio degli anni '60 l'incidenza del Risorgimento nel discorso pubblico, il $D B I$ poteva rilanciare uno sforzo scientificamente più ragionato: anche attraverso collaboratori quali, tra i tanti, Ettore Passerin d'Entreves (Cavour), Ernesto Sestan (Cattaneo), Armando Saitta (Buonarroti), Franco Della Peruta (i fratelli Bandiera), esso poté dare voce ai nuovi indirizzi di ricerca, alle posizioni e ai dibattiti storiografici accesi che nel dopoguerra avevano rivisitato le origini dello stato nazionale.

Su tutti questi livelli di riflessione, Casalena sosta con esemplificazioni ben distribuite e argomentazioni convincenti, che incrociano le fonti e la storiografia con notevole sensibilità e capacità di sintesi. D'altra parte, l'impianto del volume, centrato su ambizioni, tensioni interne, metamorfosi di cui quelle imprese editoriali sono intessute, finisce per relegare sullo sfondo una verifica, anche parziale, dell'accoglienza che ebbero nel dibattito scientifico e pubblico. Volgendo lo sguardo ai destinatari, viene da chiedersi fino a che punto, con quali risultati e quali limiti, le voci dei grandi dizionari abbiano modellato una

${ }^{21}$ Si pensi agli agili medaglioni biografici della «Centuria di ferro»: cfr. M. Baioni, "Gli italiani sanno morire». Una collana storica per le guerre del fascismo, «Contemporanea», 18 (2015), n. 2, pp. 245-66.

${ }^{22}$ Sempre valide al proposito le pagine di P.G. Zunino, L'ideologia del fascismo. Miti, credenze e valori nella stabilizzazione del regime, il Mulino, Bologna 1985. Alcune indicazioni recenti, seppur schematiche, sono in F. Albano, Cento anni di padri della patria 1848-1948, Carocci, Torino-Roma 2017. 
immagine del passato risorgimentale che sia possibile registrare negli echi del loro percorso travagliato. Il problema si pone evidentemente anche in termini di quantità e qualità della ricezione. Una cosa sono i libri e gli opuscoli biografici che, entrando nel circuito del mercato editoriale, riscuotono una fortuna misurabile dalle vendite o dalle accoglienze presso la stampa. Nel caso dei dizionari il riscontro, va da sé, è decisamente più complicato e rarefatto: nella veste di «miti di consultazione» (p. 84), la loro circolazione finisce per essere limitata alle biblioteche e agli istituti culturali, con un uso che tende ad essere prevalentemente specialistico, benché non vadano sottovalutate le eventuali ricadute sul versante scolastico. Ma anche sugli aspetti che attengono alla diffusione e alla ricezione della cultura biografica, l'importante libro di Casalena costituisce uno stimolante punto di partenza per nuove esplorazioni di ricerca. 